Z. Klin. Chem. Klin. Biochem.

10. Jg. 1972, S. $326-328$

\title{
Veränderte Fettsäurezusammensetzung des Subcutanfettes bei Ratten mit hypothalamischer Fettsucht
}

\author{
Von E. Scharrer und Renate Schubert \\ Institute für Tierphysiologie und Ernäbrungsphysiologie \\ (Vorstände: Prof. DDr. DDr. h. c.J. Brüggemann, Prof. Dr. Dr. J. Tienvs) der Universität München
}

(Eingegangen am 28. Februar 1972)

Das Fettsäuremuster des Subcutanfettes hypothalamisch fettsüchtiger Ratten wurde untersucht. Im Vergleich zu normalen Kontrollratten wurde bei Ratten mit hypothalamischer Fettsucht eine signifikante Zunahme des Anteils von 16:0-, 16:1- und 18:1-Fettsäuren, sowie eine signifikante Verringerung des Gehalts an 18:2- und 20:3-Fettsäuren ermittelt. Z̈ehntägiger Futterentzug führte bei den fettsüchtigen Tieren zu keiner Veränderung des Fettsäuremusters.

\section{Alterations in the fatty acid composition of subcutaneous fat in rats with bypothalamic obesity}

The fatty acid composition of the subcutaneous fat was studied in rats with hypothalamic obesity. Compared with normal control rats, the rats with hypothalamic obesity showed a significant increase in the proportion of 16:0,16:1 und 18:1 fatty acids and a significant decrease in the content of the 18:2 and 20:3 fatty acids. Starvation for ten days caused no change in the fatty acid pattern of the rats with hypothalamic obesity.

Bilaterale hypothalamische Läsionen im Bereich des Nucleus ventromedialis verursachen bei Ratten $(1,2)$ und verschiedenen anderen Spezies (3-8) Hyperphagie und Fettsucht. Die Fettsucht scheint dabei nicht ausschließlich durch Hyperphagie zustandezukommen (9).

Haessler und Crawford $(10,11)$ haben neuerdings typische Veränderungen in der Fettsäurezusammensetzung des Depotfetts hypothalamisch fettsüchtiger Ratten beschrieben. Eigene diesbezügliche Untersuchungen, über die nachfolgend berichtet wird, erbrachten zum Teil erhebliche Abweichungen von den Befunden dieser Autoren. Das Fettsäuremuster des Depotfettes normaler Ratten.(12, 13) und Mäuse (14) sowie von Mäusen mit hyperglycämischer Fettsucht (14) verändert sich anscheinend während einer längeren Fastenperiode. In unseren Untersuchungen wurde daher zusätzlich geprüft, inwieweit dies auch für Ratten mit hypothalamischer Fettsucht zutrifft.

\section{Material und Methoden}

\section{Versucbstiere}

Als Versuchstiere fanden erwachsene weibliche Sprague-DawleyRatten Verwendung, die mit einem handelsüblichen Rattenfutter ${ }^{1}$ ) (Rohfett: $4 \%=11 \mathrm{Kal} \%$, Rohprotein: $20 \%=26 \mathrm{Kal} \%$ ) ad libitum gefüttert wurden. Für den Fettanteil des Futters wurde das in Tabelle 1 angegebene Fettsäuremuster ermittelt.

Die Tiere waren in Einzelkäfigen untergebracht und wurden bei einer Raumtemperatur von $24 \pm 1^{\circ}$ gehalten. Sie wogen zum Zeitpunkt der Operation (s. u.) durchschnittlich 230 g. $21 / 2$ Monate nach der Operation betrug das Durchschnittsgewicht der für die Versuche ausgewählten operierten Tiere $493 \mathrm{~g}$ (407-665 g). Die Kontrolltiere wiesen zu diesem Zeitpunkt ein Durchschnittsgewicht von $250 \mathrm{~g}$ auf. Normale weibliche Sprague-Dawley'Ratten

1) Altromin $\mathrm{GmbH}$, Lage/Lippe.
Tab. 1

Fettsäuremuster des Fettanteils des Rattenfutters

\begin{tabular}{lr}
$\begin{array}{c}\text { Fettsäure } \\
(\text { C: } \Delta)\end{array}$ & $\begin{array}{c}\text { Anteil } \\
(\%)\end{array}$ \\
\hline $14: 0$ & 2,4 \\
$16: 0$ & 18,5 \\
$16: 1$ & 3,0 \\
$18: 0$ & 5,4 \\
$18: 1$ & 29,0 \\
$18: 2$ & 29.0 \\
$18: 3$ & 2,5 \\
$20: 1$ & 1,6 \\
$20: 2+21: 0$ & 1,7 \\
$20: 3$ & 1,5 \\
$20: 5$ & 2,3 \\
\hline
\end{tabular}

werden im allgemeinen nicht schwerer als $300 \mathrm{~g}$. Während eines 10-tägigen Futterentzuges verringerte sich das Durchschnittsgewicht der fettsüchtigen Tiere von 493 auf $382 \mathrm{~g}$.

\section{Erzeugung der Läsionen}

Zur Erzeugung der bilateralen hypothalamischen Läsionen (15) im Bereich des Nucleus ventromedialis wurde ein stereotaktisches Instrument nach Stellar der Firma C. $H$. Stoelting Co. verwendet. Die Tiere wurden mit Equi-Thesin $\left.{ }^{2}\right)(3 \mathrm{ml} / \mathrm{kg}$ Körpergewicht, intraperitoneal) narkotisiert. Anschließend wurde nach Trepanation des Schädels bilateral eine, abgesehen von der Spitze, mit Teflon isolierte Elektrode aus rostfreiem Stahl gemäß den stereotaktischen Koordinaten von DE. GROot (16) sowie BERNARDIS und SkELTON (17) in den Hypothalamus eingeführt. Folgende Koordinaten (in $\mathrm{mm}$ ) wurden verwendet: 5,9 anterior; 0,65 lateral; 1,0 über dem os sphenoides. Zur Erzeugung der Läsionen wurde ein Gleichstrom von $2 \mathrm{~mA}$ für $15 \mathrm{~s}$ durch die nicht isolierte Elektrodenspitze geschickt. Der Erfolg der Operation wurde aufgrund der Gewichtsentwicklung beurteilt (s. o.).

2) Equi-Thesin $=4,25 \mathrm{~g}$ Chloralhydrat; 0,97g Pentobarbital, 2,13 $\mathrm{g}$ Magnesiumsulfat, 42,8\% Propylenglycol, $11,5 \%$ Athanol in $100 \mathrm{ml}$ wäßr. Lösung. Jensen-Salsbery Laboratories, Division of Richardson Merrel, Inc., Kansas City, Mo. 
Tab. 2

Fettsäuremuster des Depotfettes von Ratten. Angegeben ist die Zusammensetzung in o; $(\bar{x} \pm s)$. Die Signifikanz wurde mit dem $t$ Test ermittelt

Eigene Ergebnisse (Subcutanfett)

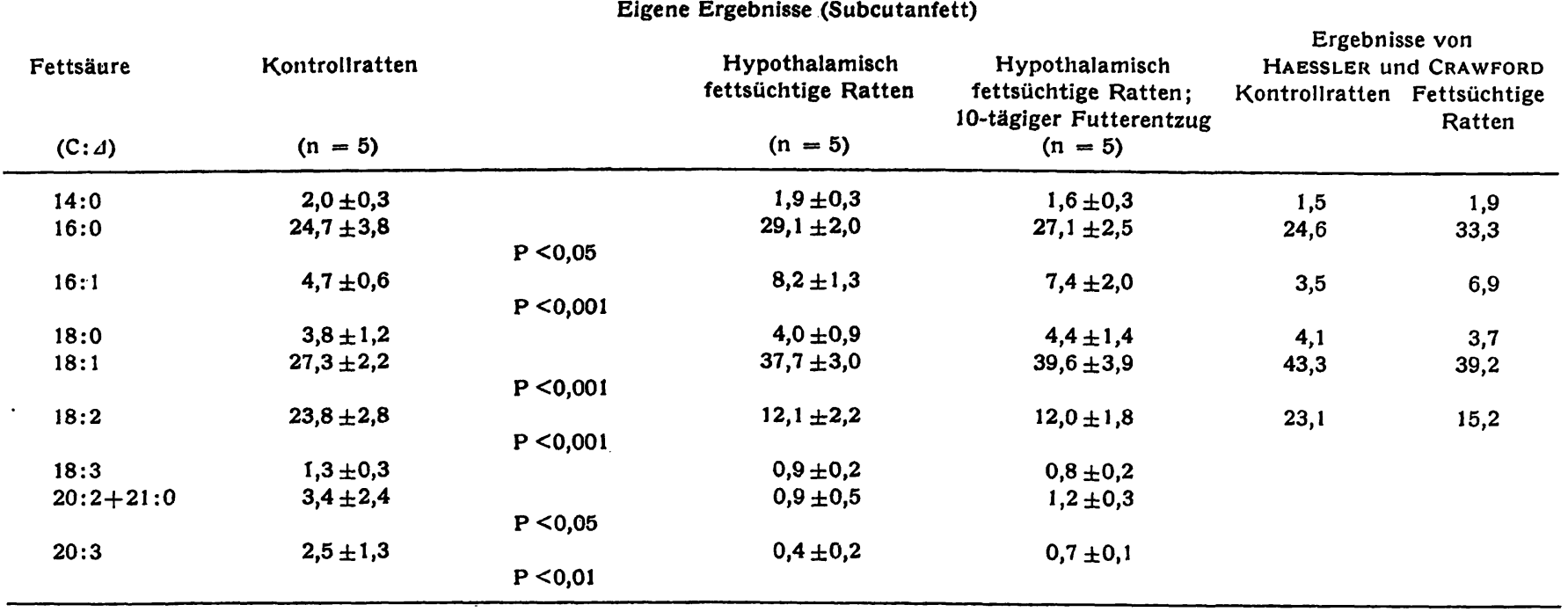

\section{Probenabme}

Die Entnahme der Subcutanfett-Proben erfolgte unter Athernarkose seitlich am Abdomen. Die Proben wurden bis zur Aufarbeitung bei $-20^{\circ}$ aufbewahrt.

\section{Aufarbeitung der Proben}

Die Aufarbeitung der Proben zur gaschromatographischen Bestimmung der Fettsäuren wurde an anderer Stelle ausführlich beschrieben (18).

Nach Verseifung der Fettproben und Verdünnen mit Wasser wurden die Seifen durch Ansäuern mit konz. $\mathrm{HCl}$ auf $\mathrm{pH} 3$ in die Fettsäuren überführt und diese mit Petroläther (Kp 30-50 $)$ extrahiert. Die Fettsäuren wurden mit Bortrifluoriddiäthylätherat und Methanol in ihre Methylester überführt.

\section{Gaschromatographie}

Die gaschromatographische Analyse der Fettsäuremethylester (18) erfolgte mit dem Fraktometer F 7 der Fa. Perkin Elmer mit angeschlossenem Hitachikompensationsschreiber (Stahlsäule $2 \mathrm{~m} \times$ $2,5 \mathrm{~mm}, 10 \%$ Äthylenglykolsuccinatmethylsilikon (EGSS-X) auf Gaschrom P (100-120 mesh), Hitzdrahtdetektor, Helium $60 \mathrm{ml} /$ min, Arbeitstemperatur $190^{\circ}$, Einspritzblock $300^{\circ}$, Probenvolumen $0,5 \mu \mathrm{l})$.

Fettsäuren, deren prozentualer Anteil bei beiden Tiergruppen unter $1 \%$ lag, werden bei den Ergebnissen nicht angegeben.

\section{Ergebnisse}

In Tabelle 2 wird das Fettsäuremuster des Subçutanfettes der Normaltiere sowie der fettsüchtigen Tiere vor und nach dem 10-tägigen Futterentzug wiedergegeben. Es fällt auf, daß der Anteil der Fettsäure 18:2 im Subcutanfett der Kontrollratten den entsprechenden Wert der fettsüchtigen Ratten um etwa das Doppelte übertrifft, während sich für die Fettsäuren 16:0, 16:1 und 18:1 bei den fettsüchtigen Tieren signifikant höhere Werte ergaben.

Für die Fettsäuren 20:2 (+21:0) und 20:3 gilt das Umgekehrte. Der Futterentzug wirkte sich, wie ebenfalls aus der Tabelle hervorgeht, auf das Fettsäuremuster des Subcutanfettes der fettsüchtigen Tiere nicht aus. Normaltiere wurden in diese Untersuchungen nicht miteinbezogen.

\section{Diskussion}

Haessler und Crawford $(10,11)$ beobachteten am epididymalen Fettgewebe hypothalamisch fettsüchtiger Ratten im Vergleich zu normalen Kontrolltieren eine deutliche Zunahme des Anteils von 16:0- und 16:1sowie eine markante Abnahme des Anteils von 18:2Fettsäuren. Dies deckt sich mit unseren am Subcutanfett erhaltenen Ergebnissen. Jedoch wurde in unseren Untersuchungen bei den Tieren mit hypothalamischer Fettsucht gegenüber den Kontrolltieren zusätzlich eine signifikante Erhöhung des Anteils von 18:1- sowie eine signifikante Verringerung des Anteils von 20:2(十 21:0)- und 20:3-Fettsäuren ermittelt. Zum besseren Vergleich werden in Tabelle 2 die von HaEssLer und CRAWFORD ermittelten Werte angegeben.

Es zeigt sich, daß in den Versuchen dieser Autoren das epididymale Fettgewebe der fettsüchtigen Tiere im Vergleich zu den Kontrolltieren sogar einen etwas niedrigeren Anteil von 18:1-Fettsäuren aufwies. Angaben über den prozentualen Gehalt des Depotfettes an mehrfach ungesättigten langkettigen Fettsäuren werden von diesen Autoren nicht gemacht.

Für die nach Erzeugung der hypothalamischen Läsionen auftretende Hyperlipogenese (19) scheint somit nach unseren Befunden vor allem eine gesteigerte Akkumulation der Palmitinsäure sowie der Monoensäuren im Depotfett charakteristisch zu sein.

Das sich in unseren Untersuchungen im Vergleich zu den Befunden von Haessler und Crawford $(10,11)$ manifestierende andersartige Verhalten der 18:1-Fettsäure (s. o.) könnte darauf zurückzuführen sein, daß, wie die entsprechenden Werte bei den Kontrollratten erkennen lassen (s. o.), der 18:1-Anteil im Depotfett unserer Versuchstiere zum Zeitpunkt der Erzeugung der hypothalamischen Läsionen erheblich niedriger war. Dies dürfte fütterungsbedingt sein, da der Fettanteil $(6 \% \mathrm{w} / \mathrm{w})$ der von den genannten Autoren verfütterten Rattendiät einen höheren Gehalt an 18:1-Fettsäure 
(37\%) aufwies, als der Fettanteil unserer Versuchsdiät (4\% w/w Fett, Gehalt an 18:1-Fettsäure: $29 \%$ ). Möglicherweise läßt sich somit eine Erhöhung des Anteils an 18:1-Fettsäure im Depotfett als Ausdruck einer Hyperlipogenese nur dann nachweisen, wenn der Ausgangswert für 18:1-Fettsäure relativ niedrig ist. Damit mag es zusammenhängen, da $\beta$ bislang auch bei verschiedenen anderen Arten experimenteller Fettsucht im Depotfett kein erhöhter Anteil an 18:1-Fettsäure nachgewiesen werden konnte $(10,20)$.

Die trotz der erhöhten Aufnahme von 18:2-Fettsäure (Hyperphagie!) zu verzeichnende drastische Verringerung des Anteils von 18:2-Fettsäure im Depotfett der fettsüchtigen Tiere läßt sich auf der Basis der gesteigerten Lipogenese erklären, da ja die aus Kohlenhydraten gebildeten Fettsäuren frei von Linolsäure sind.

Auffällig ist der relativ hohe Anteil an 20:2- $(+21: 0)-$ und 20:3-Fettsäuren im Subcutanfett der Kontrolltiere. Dies steht in Einklang mit Befunden anderer Autoren, die am subcutanen Fettgewebe (21) bzw. an den Hautlipiden (22) von Ferkeln (21) und Kälbern (22) erhoben wurden.

Det im Vergleich zu den Kontrolltieren geringe Anteil an 20:2- (+ 21:0-) und 20:3-Fettsäuren im Depotfett der operierten Tiere deutet darauf hin, daß sich die Hyperlipogenese auf diese Fettsäuren nicht erstreckt. Ein zehntägiger Futterentzug wirkte sich auf das Fettsäuremuster des Subcutanfettes der fettsüchtigen Ratten nicht aus. Dies zeigt, $\mathrm{da} ß$ unter den angegebenen Versuchsbedingungen die energetische Verwertung der einzelnen Fettsäuren proportional zu ihren im Depotfett vorliegenden Anteilen erfolgt, was mit Befunden anderer Autoren $(12,13,14)$ nicht vereinbar ist.

Diese Autoren ermittelten im Depotfett normaler Ratten (12, 13) und Mäuse (14) nach mehrtägigem Futterentzug eine Verringerung des Anteils von 16:0und 16:1-Fettsäuren (12) sowrie eine Zunahme des Anteils von 18:0- (12), 18:1- $(12,13,14)$ und 18:2Fettsäuren (12). Bei Mäusen mit hyperglycämischer Fettsucht erhöhte sich unter diesen Umständen lediglich der Anteil von 18:2-Fettsäure (14). Auch andere Autoren berichten über eine erhöhte Persistenz von 18:2-Fettsäure im Depotfett normaler Ratten und Mäuse $(20,23,24,25)$, wogegen sich bei Mäusen mit Goldthiogluccose-Fettsucht 3-tägiger partieller Futterentzug auf die Fettsäurezusammensetzung des Depotfetts ebenfalls nicht auswirkte (20).

Mit Unterstützung der Deutschen Forschungsgemeinschaft.

\section{Literatur}

1. Hetherington, A. W. und S. W. Ranson, Anat. Rec. 78,149 (1940). - 2. Hetherington, A. W. und S. W. Ranson, Amer. J. Physiol. 136, 609 (1942). - 3. ANAND, B. K. und J. R. BroneCK, Yale J. Biol. Med. 24, 123 (1951). - 4. MAYER, J., R. G., French, C. Y. Zighera und R. J. Barnett, Amer. J. Physiol. 182, 75 (1955). - 5. Balinska, H., Acta Biol. Exp. 23, 33 (1963). 6. Hamilton, C. L. und J. R. Brobeck, J. Comp. Physiol. Psychol. 57, 271 (1964). - 7. Barle, C. A., J. MaYer und A. W. Mahoney, J. Dairy Sci. 52, 101 (1969). - 8. Auffrey, P., Ann. Biol. anim. Bioch. Biophys. 9, 513 (1969). - 9. HAN, P. W. und L. A. Frohman, Amer. J. Physiol. 219, 1632 (1970). - 10. Haessler, H. A. und J. D. Crawford, Ann. N. Y. Acad. Sci. 131, 476 (1965). - 11. Haessler, H. A. und J. D. Crawford, Amer. J. Physiol. 213, 255 (1967). - 12. Hollenberg, C. H. und D. E. Douglas, Nature 153, 1074 (1962). - 13. Suzukr, M. und T. Koyanagi, J. Jap. Soc. Food Nutr. 21, 171 (1968). 14. Stein, J., J. Anderson und G. Hollifield, Metabolism 16,
758 (1967). - 15. Scharrer, E., C. A. Baile und J. Mayer' Amer. J. Physiol. 218, 400 (1970). - 16. DE. Groot, The Rat Forebrain in Stereotactic Coordinates. Amsterdam (1959). N. V. Noord Hollandsche Uitgevers Maatschappij. - 17. BerNARDIs, L. L. und F. R. Skelton, Amer. J. Anat. 116, 69 (1965). - 18. SChubert, R., Dissertation, Univ. München (1969). - 19. Bates, M. W., C. Zomzely und J. Mayer, Amer. J. Physiol. 181, 187 (1955). - 20. Soyka, L. F., H. A. HAESSLER und J. D. CRAwFord, Amer. J. Physiol. 217, 1088 (1969). - 21. Molnar, S., Z. Tierphysiol., Tierern., Futtermittelkunde 24, 330 (1968/69). - 22. Ter Meulen, U., S. Molnar und H. Neumann, Z. Tierphysiol., Tierern., Futtermittelkunde 26, 206 (1970). - 23. WAGNER, H., E. Seelig und K. Bernhard, Hoppe Seyler's Ż. physiol. Chem. 312, 104 (1958). - 24. JacQuot, R., Y. Abraham, R. Reveux, M. Brunaud und J. Tremolreres, Nutr. Dieta 1, 221 (1959). 25. Raulin, J. und M. LaunaY, C. R. Acad. Sci. 258, 6542 (1964).

Priv.-Doz. Dr. E. Scharrer Institut $\mathrm{f}$. Tierphysiologie 8000 München 22

Veterinärstr. 13 


\section{BIOCHIMIE}

Edité par la Société de Chimie Biologique

tel est le titrc

sous lequel paraittra à partir de 1971

le „BULLETIN DE LA SOCIÉTÉ

DE CHIMIE BIOLOGIQUE“"

\section{SECRÉTARIAT}

de la Société de Chimie Biologique

J.P. EBEL, Secrétaire Général (Relations Extérieures)

R. PERLES, Secrétaire Général

\section{REDACTION}

F. GROS, Secrétaire scientifique

F. PERCHERON, Secrétaire à la Publication

J. NUNEZ, Secrétaire à l'Information

Y. RAOUL, Secrétaire à l'Edition

\section{SECRETARIAT et REDACTION:}

4 Avenue de l'Observatoire, PARIS $6^{\circ}$

12 FASCICULES

ABONNEMENTS: FRANCE et ZONE FRANC: 150 ffrcs

BELGIQUE: $1.687,-$ frcs

AUTRES PAYS : $186,-$ ffrcs

MASSON et Cie, Editeurs

120 Boulevard St Germain

PARIS 6ème

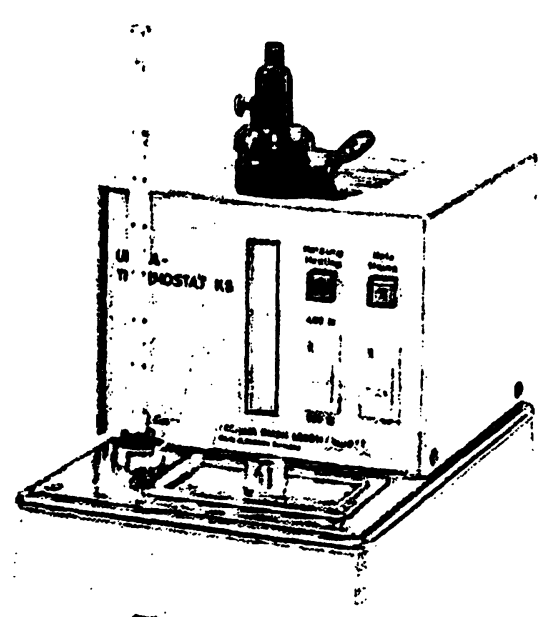

COlOp

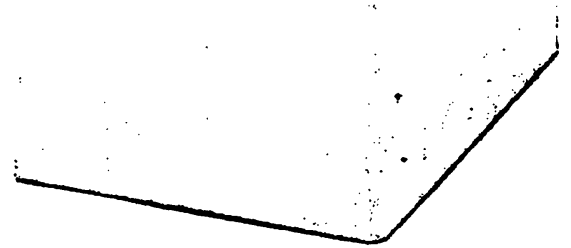

\section{Colora-}

\section{Ultra-Thermostat K 5}

Kleinthermostat modernster Konstruktion für Temperierungen im Bad und im Umwälzverfahren. K 5 mit Druckpumpe, K 5 DS mit Druck- und Saugpumpe. Lagerfreie Umwälzpumpe hoher Leistung. Funkentstörtes Elektronikrelais. Temperaturkon$\operatorname{stanz} \pm 0,02^{\circ} \mathrm{C}$. Hochwertiges Material in erstklassiger Verarbeitung. MetallteiledesTemperiersystemsaus rostfreiem Edelstahl. Ein zuverlässiges Gerät zu günstigem Preis.

\section{Colora Messtechnik GmbH} 7073 Lorch/Württ., Postfach 5 T (07172) 6041, FS 07-248 886

Technische Büros (Verkauf und Kundendienst): 1000 Berlin 30, Kurfürstenstraße 84, T 2615200 2000 Hamburg 19, Osterstraße 63, T 4911034 , FS 02-12 947 3000 Hannover, An der Tiefenriede 45, T 884500 4000 Düsseldorf, Kronprinzenstr. 62, T 3201 64, FS 08-587 253 6000 Frankfurt a.M., Rõderbergweg 4-6,T446031, FS 04-11 216 8000 München 19, Dachauer Straße 175, T 193858

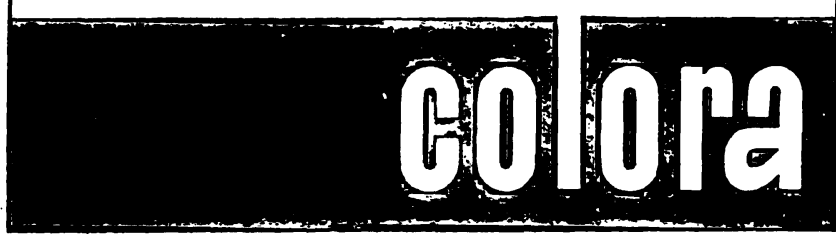




\section{Lithium, Clinical and Biological Aspects}

Reprint from International Pharmacopsychiatry, Vol. 5, Nos. 2-4, 1971

Editor: F. A.Freyban (New York, N. Y.)

192 p., 29 fig., 37 tab., 1971

SFr. 49.20 / US $\$ 13.80 / \mathrm{DM} 49,20 / £ 5.43$

ISBN 3-8055-1277-5

Editorial, Lithium: Some Critical Considerations - Long-Term Drug Therapy in Affective Disorders. Theoretical and Methodological Issues in Current Research on Lithium - Studies on the Course of Recurrent Endogenous Affective Disorders - Clinical Controversies and Theoretical Mode of Action of Lithium Carbonate - The Course of Recurrent Depressive Illness in Selected Patients from a Defined Population - Lithium Carbonate: A Drug of Limited Efficacy in the Prevention of ManicDepressive Disease - Treatment of Mood Disorders with Lithium Carbonate - Ergebnisse elektroenzephalographischer Untersuchungen zur Lithiumwirkung (The results of electroencephalographic research and the effect of lithium) - Lithium Drop-Outs. A Follow-Up Study of Patients who Discontinued Prophylactic Treatment - Neurotoxicity with Lithium: Differential Drug Responsiveness Sustained-Release Lithium Carbonate - Indications of Lithium Salts in Psychosomatic Medicine Psychosocial Profiles of Patients on Lithium Treatment - Antiaggressive Effect of Lithium Salts in Severe Mentally Retarded Adolescents - Structural Basis of Lithium Psychopharmacology. Extracellular Ground Substance of Rat Brain - Complications or Coincidences During Lithium Therapy. A Review of Several Cases - Die prophylaktische Wirkung von Lithiumsalzen und Schilddrüsenhormonen auf Manifestationen des manisch-depressiven Krankheitsgeschehens und der Legierungspsychosen. Klinische und experimentelle Ergebnisse (Prophylaxis of manicdepression and schizoaffective disorders by lithium salts and thyroid hormones. Clinical and experimental results) - Certain Circadian Rhythms in Manic-Depressives and their Response to Lithium - Lithium-Induced Polyuria in Rats - Effect of Lithium and Other Psychopharmaca on Rat Electrolyte Metabolism - Zur Stellung des Lithiums in der Gruppe der Antidepressiva in der Behandlung von akuten endogenen und Involutionsdepressionen (The position of lithium among antidepressants in the treatment of acute phase of endogenous and involutional depressions) - Lithium Effects on Calcium Metabolism in Rats.

The purpose of this reprint is to provide the reader with important investigative information on the clinical use and the mode of action of lithium. The selections have been contributed from some of the most important investigators in the field, lending an international scope to this work which ranges from clinical studies to basic laboratory findings. Many of the reported results convey a new picture of the efficacy of lithium in the treatment of psychiatric disorders, and it is in this sense that the reprint is both provocative and stimulating for scientists as well as practitioners.

\section{S. Karger}

Basel · München $\cdot$ Paris $\cdot$ London $\cdot$ New York $\cdot$ Sydney 